\title{
High-density reflection spectroscopy: I. A case study of GX 339-4
}

\author{
Jiachen Jiang ${ }^{\oplus},{ }^{1 \star}$ Andrew C. Fabian ${ }^{\oplus}, 1$ Jingyi Wang, ${ }^{2}$ Dominic J. Walton ${ }^{\oplus},{ }^{1}$ \\ Javier A. García, ${ }^{3,4}$ Michael L. Parker ${ }^{\oplus},{ }^{5}$ James F. Steiner ${ }^{2}$ and John A. Tomsick ${ }^{6}$ \\ ${ }^{1}$ Institute of Astronomy, University of Cambridge, Madingley Road, Cambridge CB3 OHA, UK \\ ${ }^{2}$ MIT Kavli Institute for Astrophysics and Space Research, MIT, 70 Vassar Street, Cambridge, MA 02139, USA \\ ${ }^{3}$ Cahill Center for Astronomy and Astrophysics, California Institute of Technology, Pasadena, CA 91125, USA \\ ${ }^{4}$ Dr. Karl Remeis-Observatory and Erlangen Centre for Astroparticle Physics, Sternwartstr. 7, D-96049 Bamberg, Germany \\ ${ }^{5}$ European Space Agency (ESA), European Space Astronomy Centre (ESAC), E-28691 Villanueva de la Cañada, Spain \\ ${ }^{6}$ Space Sciences Laboratory, 7 Gauss Way, University of California, Berkeley, CA 94720, USA
}

Accepted 2019 January 6. Received 2019 January 6; in original form 2018 November 26

\begin{abstract}
We present a broad-band spectral analysis of the black hole binary GX 339-4 with NUSTAR and Swift using high-density reflection model. The observations were taken when the source was in low-flux (LF) hard states during the outbursts in 2013 and 2015, and in a very high-flux (HF) soft state in 2015. The high-density reflection model can explain its LF spectra with no requirement for an additional low temperature thermal component. This model enables us to constrain the density in the disc surface of GX 339-4 in different flux states. The disc density in the LF state is $\log \left(n_{\mathrm{e}} / \mathrm{cm}^{-3}\right) \approx 21,100$ times higher than the density in the HF state $\left(\log \left(n_{\mathrm{e}} / \mathrm{cm}^{-3}\right)=18.93_{-0.16}^{+0.12}\right)$. A close-to-solar iron abundance is obtained by modelling the $\mathrm{LF}$ and $\mathrm{HF}$ broad-band spectra with variable density reflection model $\left(Z_{\mathrm{Fe}}=1.50_{-0.04}^{+0.12} \mathrm{Z}_{\odot}\right.$ and $Z_{\mathrm{Fe}}=1.05_{-0.15}^{+0.17} \mathrm{Z}_{\odot}$, respectively).
\end{abstract}

Key words: accretion, accretion discs - X-rays: binaries - X-rays: individual (GX 339-4).

\section{INTRODUCTION}

The primary X-ray spectra from black holes $(\mathrm{BHs})$ can be described by a power-law continuum, which originates from a high temperature compact structure external to the $\mathrm{BH}$ accretion disc. This high temperature compact structure is called the corona. The interaction between the primary power-law photons and the disc top layer can produce both emission, including fluorescence lines and recombination continuum, and absorption edges. These features are referred to as the disc reflection spectrum (e.g. George \& Fabian 1991; García \& Kallman 2010). The disc reflection spectrum is highly affected by relativistic effects, such as Doppler effect and gravitational redshift, due to the strong gravitational field in the vicinity of BHs (e.g. Reynolds \& Nowak 2003). For example, relativistic blurred $\mathrm{Fe} \mathrm{K} \alpha$ emission line features have been detected in reflection spectra of both active galactic nuclei (AGNs; e.g. MCG-6-30-15, Tanaka et al. 1995) and Galactic BH X-ray binary (XRB) sources (e.g. Cyg X-1, Barr, White \& Page 1985). Relativistic reflection spectra can provide information on the disc-corona geometry, such as the coronal region size and the disc inner radius. By comparing relativistic reflection spectra in different observations, we can investigate changes of the inner accretion processes through the evolution of the X-ray flux states

^E-mail: jj447@cam.ac.uk in both highly variable AGNs (e.g. Mrk 335, IRAS 13224-3809, Parker et al. 2014; Jiang et al. 2018) and XRBs that show different flux states (e.g. XTE J1650-500, Reis et al. 2013).

The existence of two different flux states in XRB was first realized in the X-ray emission of the XRB Cyg X-1 (Oda et al. 1971). Its $\mathrm{X}$-ray spectrum can change from a soft spectrum featured by a strong thermal component to a hard spectrum featured by a strong disc reflection component. The soft state, which is also characterized by no radio detection, is identified as the 'high' flux (HF) state and the hard state with associated radio detection is identified as the 'low' flux (LF) state due to the large flux variation during the transition. Measurements in the HF soft states of BH XRB offer good evidence that the accretion disc is extended to the innermost stable circular orbit (ISCO; e.g. LMC X-3, Steiner et al. 2010). Most of the spin measurements of soft states are based on the assumption that the inner radius is located at ISCO (e.g. Gou et al. 2014; Walton et al. 2016). In the LF hard state, the disc is predicted to be truncated at a large radius and replaced by an advective flow at small radii (Esin, McClintock \& Narayan 1997; Narayan 2005). Although there is evidence that the disc is truncated as measured by reflection spectroscopy at X-ray luminosities $L_{\mathrm{X}} \approx 0.1$ per cent $L_{\text {Edd }}$ (Narayan \& McClintock 2008; Tomsick et al. 2009), there is a substantial debate whether the disc is truncated in the intermediate flux hard state due to different spectral modelling or instrumental pile-up effects (see the discussion in Wang-Ji et al. 2018). 
A common result obtained by reflection modelling of BH X-ray spectra is high iron abundance compared to solar. For example, Walton et al. (2016) found a value of $Z_{\mathrm{Fe}} \approx 4 \mathrm{Z}_{\odot}$ in Cyg X-1 and Parker et al. (2015) obtained $Z_{\mathrm{Fe}} \approx 4.7 \mathrm{Z}_{\odot}$ in the same source. Similarly, an iron abundance of $Z_{\mathrm{Fe}} \approx 2-5 \mathrm{Z}_{\odot}$ is required for another BH XRB V404 Cyg (Walton et al. 2017). Such a high iron abundance has been commonly seen in AGNs as well (e.g. Chiang et al. 2015; Parker, Miller \& Fabian 2018). Wang et al. (2012) found that the metallicity of the outflows in different quasars can vary between 1.7 and $6.9 Z_{\odot}$. Reynolds et al. (2012) suggested that the radiation-pressure dominance of the inner disc may enhance the iron abundances. However, radiative levitation effects make predictions for a change of the inner disc iron abundance, which is difficult to be observed in AGNs due to their longer dynamical time-scales.

Another possible explanation for the high iron abundances is high-density reflection. Most versions of available disc reflection models assume a constant electron density $n_{\mathrm{e}}=10^{15} \mathrm{~cm}^{-3}$ for the top layer of the $\mathrm{BH}$ accretion disc, which is appropriate for very massive supermassive BHs in AGNs (e.g. $M_{\mathrm{BH}}>10^{8} \mathrm{M}_{\odot}$ ). For example, an upper limit of $n_{\mathrm{e}}<10^{15.3} \mathrm{~cm}^{-3}$ is obtained in Seyfert 1 galaxy $1 \mathrm{H} 0419-577\left(M_{\mathrm{BH}} \approx 1.3 \times 10^{8} \mathrm{M}_{\odot}\right.$, Grupe et al. 2010 ) by fitting its $X M M-N e w t o n$ spectra with variable density reflection model (Jiang et al. 2019). At higher electron density, the free-free process becomes more important in constraining lowenergy photons, increasing the temperature of the top layer of the disc, and thus turning the reflected emissions below $1 \mathrm{keV}$ into a blackbody shaped spectrum (Ross \& Fabian 2007; García et al. 2016). Such a model can potentially relieve the very high iron abundance required in previous reflection spectral modelling. For instance, Tomsick et al. (2018) obtained an electron density of $n_{\mathrm{e}} \approx 3 \times 10^{20} \mathrm{~cm}^{-3}$ by fitting the Cyg X-1 intermediate flux state spectra with the high electron density reflection model. Although the iron abundance was fixed at the solar value during the spectral fitting, the model successfully explains the spectra. Jiang et al. (2018) fitted the narrow-line Seyfert 1 galaxy IRAS 13224 - 3809 spectra and obtained an electron density of $n_{\mathrm{e}}>10^{19.7} \mathrm{~cm}^{-3}$ with an iron abundance of $Z_{\mathrm{Fe}} \approx 5 \mathrm{Z}_{\odot}$, which is significantly lower than the previous results $Z_{\mathrm{Fe}} \approx 20 \mathrm{Z}_{\odot}$ and closer to the iron abundance measured in the ultra-fast outflow of the same source.

Higher densities may also potentially explain the weak low temperature thermal component found in the LF state of the XRBs (e.g. Reis et al. 2008; Wang-Ji et al. 2018) and at least some of the soft excess commonly seen in Seyfert galaxies (e.g. Fabian et al. 2009; Chiang et al. 2015; Jiang et al. 2018). The inclusion of the high electron density effects significantly decreases the flux of the best-fitting blackbody component in IRAS 13224-3809 required for the spectral fitting purpose (Jiang et al. 2018). It is also interesting to note that the best-fitting flux and temperature of the blackbody component that accounts for the soft excess in IRAS $13224-3809$ show a $F \propto T^{4}$ relation, indicating a constant area origin of the soft excess emission (Chiang et al. 2015; Jiang et al. 2018).

GX 339-4 is a low-mass X-ray binary (LMXB) and shows activity in a wide range of wavelength from optical to X-ray. The mass of the central BH still remains uncertain. For example, Heida et al. (2017) obtained a $\mathrm{BH}$ mass of $2-10 \mathrm{M}_{\odot}$ by studying its nearinfrared spectrum and a mass of $>5 \mathrm{M}_{\odot}$ is obtained previously by Hynes et al. (2003a,b) and Muñoz-Darias, Casares \& Martínez-Pais (2008). The distance has been estimated to be $\approx 7 \mathrm{kpc}$ (Zdziarski et al. 2004). GX 339-4 has shown frequent outbursts and multiple X-ray observations have been taken during different spectral states of GX 339-4. In its hard state, its X-ray spectrum shows a broad iron emission line and a power-law continuum with a photon index

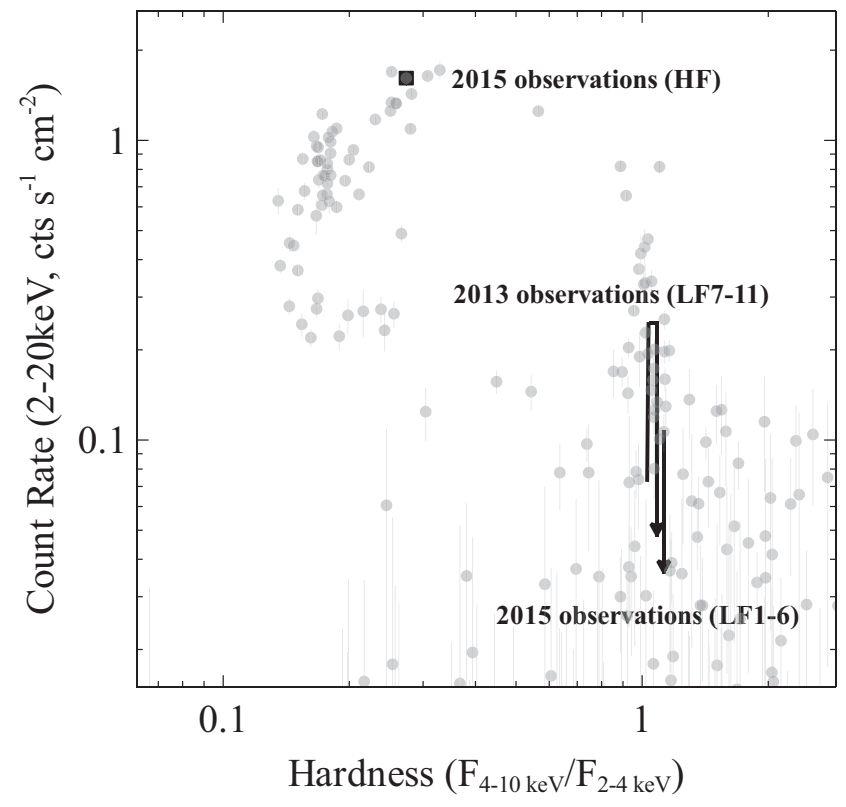

Figure 1. Weekly MAXI hardness-intensity diagram for the 2009-2018 period of GX 339-4. The black square and the arrows correspond to the dates of the HF observations and the $\operatorname{LF}(1-11)$ observations analysed in this work. The arrows show the flux change during the NUSTAR monitoring of the outbursts. NUSTAR observations were taken during the rise and decay of the outburst in 2013, and only during the decay of the outburst in 2015.

varying between $\Gamma \approx 1.5$ and 2.5 across different flux levels (Miller et al. 2004, 2006, 2008). Reis et al. (2008) presented a systematic study of its high and low hard state XMM-Newton and RXTE spectra by taking the blackbody radiation from the disc into the top layer as well as the Comptonization effects into modelling, and obtained a BH spin of $a_{*}=0.94 \pm 0.02$. More recently, Parker et al. (2016) obtained a disc iron abundance of $Z_{\mathrm{Fe}} \approx 6.6 \mathrm{Z}_{\odot}$ for the HF soft state NUSTAR and Swift spectra of GX 339-4. In this study, the disc inner radius is assumed to be located at ISCO and a BH spin of $a_{*}>0.95$ is obtained by combining disc thermal spectral and reflection spectral modelling. Later, Wang-Ji et al. (2018) found $Z_{\mathrm{Fe}} \approx 8 \mathrm{Z}_{\odot}$ for the $\mathrm{LF}$ state of the same source observed by the same instruments. Similar conclusions were found by analysing its stacked RXTE spectra at the LF states (García et al. 2015) and NUSTAR spectra during the outburst of 2013 (Fürst et al. 2015).

In this paper, we present a high-density reflection interpretation of both LF and HF state spectra of GX 339-4. The same NuSTAR and Swift spectra as in Parker et al. (2016) and Wang-Ji et al. (2018) are considered. In Section 2, we introduce the data reduction process. In Section 3, we introduce the details of high-density reflection modelling of the LF and HF spectra of GX 339-4. In Section 4, we present and discuss the final spectral fitting results. The high-density reflection modelling of AGN spectra are presented in a companion paper (Jiang in preparation).

\section{OBSERVATIONS AND DATA REDUCTION}

The weekly MAXI hardness-intensity diagram (HID) for the 2009-2018 period of GX 339-4 (Matsuoka et al. 2009) is shown in Fig. 1, showing a standard 'q-shaped' behaviour during the outbursts. GX 339-4 went through two outbursts each in 2013 and 2015. Eleven NuSTAR observations in total, each with a corresponding Swift snapshot, were triggered during these two outbursts, shown 
by the arrow in Fig. 1. The NUSTAR LF observations in 2015 were taken only during the decay of the outburst. In this work, we consider all of the NUSTAR observations taken during these two outbursts. In March 2015, GX 339-4 was detected with strong thermal and powerlaw components by Swift, suggesting strong evidence of an HF state with a combination of disc thermal component and reflection component. One NUSTAR target of opportunity observation was triggered with a simultaneous Swift snapshot. See the black square in Fig. 1 for the flux and hardness state of the source during its HF observations. A full list of observations is shown in Table 1.

\subsection{NuSTAR data reduction}

The standard pipeline NUPIPELINE V0.4.6, part of HEASOFT V6.23 package, is used to reduce the NUSTAR data. The NUSTAR calibration version V20171002 is used. We extract source spectra from circular regions with radii of 100 arcsec, and the background spectra from nearby circular regions on the same chip. The task NUPRODUCTS is used for this purpose. The $3-78 \mathrm{keV}$ band is considered for both FPMA and FPMB spectra. The spectra are grouped to have a minimum signal-to-noise ratio $(\mathrm{S} / \mathrm{N})$ of 6 and to oversample by a factor of 3 .

\subsection{Swift data reduction}

The Swift observations are processed using the standard pipeline XRTPIPELINE V0.13.3. The calibration file version used is x20171113. The LF observations taken in the WT mode are not affected by the pile-up effects. The source spectra are extracted from a circular region with a radius of 20 pixels $^{1}$ and the background spectrum spectra are extracted from an annular region with an inner radius of 90 pixels and an outer radius of 100 pixels. The LF observations taken in the PC mode are affected by the pile-up effects. By following Wang-Ji et al. (2018) where they estimated the PSF file, a circular region with a radius of 5 pixels is excluded in the centre of the source region. The $0.6-6 \mathrm{keV}$ band of all the LF Swift XRT spectra are considered. The HF observation was taken in the WT mode and was affected by pile-up effects. By following Parker et al. (2016), a circular radius of 10 pixels is excluded in the centre of the source region. The $0.6-1 \mathrm{keV}$ of the HF Swift XRT spectrum at a very HF state is ignored due to known issues of the RMF redistribution issues in the WT mode. ${ }^{2}$ The Swift XRT spectra are grouped to have a minimum $\mathrm{S} / \mathrm{N}$ of 6 and to oversample by a factor of 3 .

\section{SPECTRAL ANALYSIS}

XSPEC V12.10.0.C (Arnaud 1996) is used for spectral analysis, and $\mathrm{C}$-stat is considered in this work. The Galactic column density towards GX 339-4 remains uncertain. The value of combined $N_{\mathrm{H}_{\mathrm{I}}}$ and $N_{\mathrm{H}_{2}}$ obtained by Willingale et al. (2013) is $5.18 \times 10^{21} \mathrm{~cm}^{-2}$. However, Kalberla et al. (2005) reported a column density of $3.74 \times 10^{21} \mathrm{~cm}^{-2}$ in the Leiden/Argentine/Bonn survey. The Galactic column density values measured by different sets of broad-band X-ray spectra are different too. For example, Wang-Ji et al. (2018) obtained $\approx 4 \times 10^{21} \mathrm{~cm}^{-2}$, while Parker et al. (2016) obtained a

\footnotetext{
${ }^{1} 1$ pixel $\approx 2.36$ arcsec.

${ }^{2}$ See following website for more XRT WT mode calibration information. http://www.swift.ac.uk/analysis/xrt/digest_cal.php
}

higher value of $7.7 \pm 0.2 \times 10^{21} \mathrm{~cm}^{-2}$. We therefore fixed the Galactic column density at $3.74 \times 10^{21} \mathrm{~cm}^{-2}$ in the beginning of our analysis and allow it to vary to obtain the best-fitting value for each set of spectra. For local Galactic absorption, the tbabs model is used. The solar abundances of Wilms, Allen \& McCray (2000) are used in tbabs. An additional constant model constant has been applied to vary normalizations between the simultaneous spectra obtained by different instruments to account for calibration uncertainties.

\subsection{LF state spectral modelling}

We analyse all the LF NUSTAR observations publicly available prior to 2018 and they have discussed in Fürst et al. (2015) and Wang-Ji et al. (2018). Fig. 2 shows the ratio plots of LF1-11 spectra fitted with a Galactic absorbed power-law model obtained by fitting only the corresponding NUSTAR spectra. All the LF spectra show a broad emission line feature around $6.4 \mathrm{keV}$ with a Compton hump above $20 \mathrm{keV}$. They provide a strong evidence of a relativistic disc reflection component. By following García et al. (2015) and Wang-Ji et al. (2018), we model the features with a combination of relativistic disc reflection and a distant reflector for the narrow emission-line component. A more developed version of reflionx (Ross \& Fabian 2005) is used to model the rest-frame disc reflection spectrum. The reflionx grid allows the following free parameters: disc iron abundance $\left(Z_{\mathrm{Fe}}\right)$, disc ionization $\log (\xi)$, disc electron density $n_{\mathrm{e}}$, high-energy cut-off $\left(E_{\text {cut }}\right)$, and photon index $(\Gamma)$. All the other element abundances are fixed at the solar value (Morrison \& McCammon 1983). The ionization parameter is defined as $\xi=4 \pi F / n$, where $F$ is the total illuminating flux and $n$ is the hydrogen number density. The photon index $\Gamma$ and high-energy cut-off $E_{\text {cut }}$ are linked to the corresponding parameters of the coronal emission modelled by cutoffpl in XSPEC. A convolution model relconv (Dauser et al. 2013) is applied to the rest-frame-ionized disc reflection model reflionx to apply relativistic effects. A simple power-law-shaped emissivity profile is assumed $\left(\varepsilon \propto r^{-q}\right)$ and the emissivity index $q$ is allowed to vary during the fit. Other free parameters are the disc viewing angle $i$ and the disc inner radius $r_{\text {in }} /$ ISCO. The ionization of the distant reflector is fixed at the minimum value $\xi=10$. The other parameters of the distant reflector are linked to the corresponding parameters in the disc reflection component. The BH spin parameter $a_{*}$ is fixed at its maximum value 0.998 (Kerr 1963) to fully explore the $r_{\text {in }}$ parameter. We use cflux, a simple convolution model in XSPEC, to calculate the $1-10 \mathrm{keV}$ flux of each model component. For future reference and simplicity, we define an empirical reflection fraction as $f_{\text {refl }}=F_{\text {ref }} / F_{\mathrm{pl}}$ in the $1-10 \mathrm{keV}$ band, where $F_{\text {ref }}$ and $F_{\mathrm{pl}}$ are the flux of the disc reflection component and the coronal emission calculated by cflux. Note that this is not the same as the physically defined reflection fraction discussed by Dauser et al. (2016). The final model is tbabs * (cflux*(relconv*reflionx) + cflux*reflionx + cflux*cutoffpl) in XSPEC format. This model can fit all LF spectra successfully with no obvious residuals. For example, it offers a good fit for the LF1 spectra with $\mathrm{C}$-stat $/ v=1043.52 / 948$. A ratio plot of LF1 spectra fitted with this model is shown in the top panel of Fig. 3. The best-fitting values of some key parameters that affect the spectral modelling below $3 \mathrm{keV}$ are following: $N_{\mathrm{H}}=3.4_{-0.1}^{+0.2} \times 10^{21} \mathrm{~cm}^{-2}$, $\log (\xi / \mathrm{erg}$ $\left.\mathrm{cm} \mathrm{s}^{-1}\right)=3.18_{-0.06}^{+0.07}$, and $\log \left(n_{\mathrm{e}} / \mathrm{cm}^{-3}\right)=20.6 \pm 0.3$. Our bestfitting column density is consistent with the Galactic column density measured in Kalberla et al. (2005). 
Table 1. NUSTAR and Swift observations of GX 339-4 in 2013 and 2015. WT: window timing mode; PC: photon counting mode.

\begin{tabular}{lccccccc}
\hline Obs & NuSTAR obsID & Date & exp. (ks) & Swift obsID & Date & exp. (ks) & Mode \\
\hline HF & 80001015003 & $2015-03-04$ & 30.9 & 00081429002 & $2015-03-04$ & 1.9 & WT \\
LF1 & 80102011002 & $2015-08-28$ & 21.6 & 00032898124 & $2015-08-29$ & 1.7 & WT \\
LF2 & 80102011004 & $2015-09-02$ & 18.3 & 00032898126 & $2015-09-03$ & 2.3 & WT \\
LF3 & 80102011006 & $2015-09-07$ & 19.8 & 00032898130 & $2015-09-07$ & 2.8 & WT \\
LF4 & 80102011008 & $2015-09-12$ & 21.5 & 00081534001 & $2015-09-12$ & 2.0 & PC \\
LF5 & 80102011010 & $2015-09-17$ & 38.5 & 00032898138 & $2015-09-17$ & 2.3 & WT \\
LF6 & 80102011012 & $2015-09-30$ & 41.3 & 00081534005 & $2015-09-30$ & 2.0 & PC \\
LF7 & 80001013002 & $2013-08-11$ & 42.3 & 00032490015 & $2013-08-12$ & 1.1 & WT \\
LF8 & 80001013004 & $2013-08-16$ & 47.4 & 00080180001 & $2013-08-16$ & 1.9 & WT \\
LF9 & 80001013006 & $2013-08-24$ & 43.4 & 00080180002 & $2013-08-24$ & 1.6 & WT \\
LF10 & 80001013008 & $2013-09-03$ & 61.9 & 00032898013 & $2013-09-02$ & 2.0 & WT \\
LF11 & 80001013010 & $2013-10-16$ & 98.2 & 00032988001 & $2013-10-17$ & 9.6 & WT \\
\hline
\end{tabular}

We notice that previously the spectral modelling requires a low temperature multicolour disc thermal component diskbb $(k T=0.46 \mathrm{keV})$ when using the model with the disc electron density $n_{\mathrm{e}}$ fixed at $\log \left(n_{\mathrm{e}} / \mathrm{cm}^{-3}\right)=15$ for LF1 observation (WangJi et al. 2018). However, the normalization of this component is very low and weakly constrained. Similarly, a weak thermal component is also required in the analysis of its XMM-Newton hard state observations (Reis et al. 2008) and other earlier NuSTAR observations (Reis et al. 2013). The difference in spectral modelling may result from the following two reasons: one is the high density reflection model, where a blackbody-shaped emission arises in the soft band when the disc electron density $n_{\mathrm{e}}$ becomes higher than $10^{15} \mathrm{~cm}^{-3}$; the other is the uncertain neutral absorber column density, which was measured to be $N_{\mathrm{H}}=4.12_{-0.12}^{+0.08} \times 10^{21} \mathrm{~cm}^{-2}$ in Wang-Ji et al. (2018) and higher than our best-fitting value for the LF1 spectra.

In order to test for an additional diskbb component, we first fit the spectra with $N_{\mathrm{H}}$ fixed at the higher Galactic column density $N_{\mathrm{H}}=5.18 \times 10^{21} \mathrm{~cm}^{-2}$ obtained by Willingale et al. (2013) rather than the value from Kalberla et al. (2005). An additional diskbb component improves the fit by only $\Delta \mathrm{C}$-stat $=1.1$. See the middle panel of Fig. 3 for the corresponding ratio plot. Only an upper limit of the diskbb normalization is of $N_{\text {diskbb }}<1.5 \times 10^{5}$ found. Compared with the result in Wang-Ji et al. (2018), a lower disc inner temperature of $k T=0.24_{-0.10}^{+0.08} \mathrm{keV}$ is required in this fit. Secondly, we fit LF1 spectra with the absorber column density as a free parameter (the bottom panel of Fig. 3). A contour plot of C-stat distribution on the $N_{\mathrm{H}}$ versus $N_{\text {diskbb }}$ parameter plane is calculated by STEPPAR function in XSPEC and shown in Fig. 4. It clearly shows a strong degeneracy between the absorber column density and the normalization of the diskbb component. The fit is only improved by $\Delta \mathrm{C}$-stat $=3$ with two more free parameters after including this diskbb component. See Fig. 3 for ratio plots against different continuum models. By varying the Galactic column density, it only slightly changes the fit of the Swift XRT spectrum. Therefore, we conclude that an additional diskbb component is not necessary for LF1 spectral modelling when the disc density parameter $n_{\mathrm{e}}$ is allowed to vary. In order to visualize the spectral difference with different $n_{\mathrm{e}}$, we show the best-fitting reflection model component for LF1 in Fig. 5 in comparison with the best-fitting model for the same observation assuming $\log \left(n_{\mathrm{e}} / \mathrm{cm}^{-3}\right)=15 \mathrm{~cm}^{-3}$ in Wang-Ji et al. (2018). With a disc density as high as $\log \left(n_{\mathrm{e}} / \mathrm{cm}^{-3}\right)=20.6$, a quasi-blackbody emission arises in the soft band and accounts for the excess emission below $2 \mathrm{keV}$. Similar conclusions are found for the other sets of LF spectra. Future pile-up free high $\mathrm{S} / \mathrm{N}$ observation below $2 \mathrm{keV}$, such as from NICER, may help constrain more detailed spectral shape of LF states of GX 339-4.

So far we have achieved the best-fitting model for the LF spectra individually. We also undertake a multi-epoch spectral analysis with disc iron abundance $Z_{\mathrm{Fe}}$ and disc viewing angle $i$ linked between LF1-11 spectra. All the other parameters are allowed to vary during the fit. A table of all the best-fitting parameters is shown in Table 2. The best-fitting models and corresponding ratio plots are shown in Fig. 6. We allow the column density of the neutral absorber to vary in different epochs to investigate any variance. A slightly higher column density $\left(N_{\mathrm{H}} \approx 4.1 \times 10^{21} \mathrm{~cm}^{-2}\right)$ is required for LF6,7. The emissivity index of the relativistic reflection spectrum is weakly constrained in LF3-6 observations but largely consistent with the Newtonian value $q=3$, except for the LF1 observation. We can also confirm that the disc is not truncated at a significantly large radius, such as $r=100 r_{g}$ (Plant et al. 2015). A slight iron over abundance compared to solar is required $\left(Z_{\mathrm{Fe}}=1.5_{-0.04}^{+0.12}\right)$ for the spectral fitting. The power-law continuum is softer in the first two observations taken at higher flux levels but remains consistent at 90 per cent confidence during the rest of the decay in 2015 . The photon index in LF7-10 during the outburst in 2013 is consistent at 90 per cent confidence as well. The reflection fraction decreases with the decreasing total flux during the flux decay in 2015. This is likely caused by a receding inner disc radius at the very LF states or a change of the coronal geometry (e.g. its height above the disc). Moreover, the multi-epoch spectral analysis of all LF observations shows tentative evidence for an anticorrelation between disc density and X-ray band flux. For example, the disc density increases from $\log \left(n_{\mathrm{e}} / \mathrm{cm}^{-3}\right)=20.60_{-0.12}^{+0.23}$ in the highest flux state (LF1) to $\log \left(n_{\mathrm{e}} / \mathrm{cm}^{-3}\right)=21.45_{-0.13}^{+0.06}$ in the lowest flux state (LF6). The flux level of the cold reflection component remains consistent, indicating that this emission arises from stable material at a large radius from the central $\mathrm{BH}$. We will discuss other fitting results, such as the electron density measurements, in Section 4.

\subsection{HF state spectral modelling}

The same NuSTAR and Swift observations of GX 339-4 in an HF state analysed in Parker et al. (2016) are considered here. A ratio plot of the HF spectra fitted with a Galactic absorbed power-law model and a simple disc blackbody component diskbb is shown in the bottom panel of Fig. 2. The HF spectra show a broad emission line feature at the iron band and a Compton hump above $10 \mathrm{keV}$, indicating existence of a relativistic disc reflection component similar with all the LF 

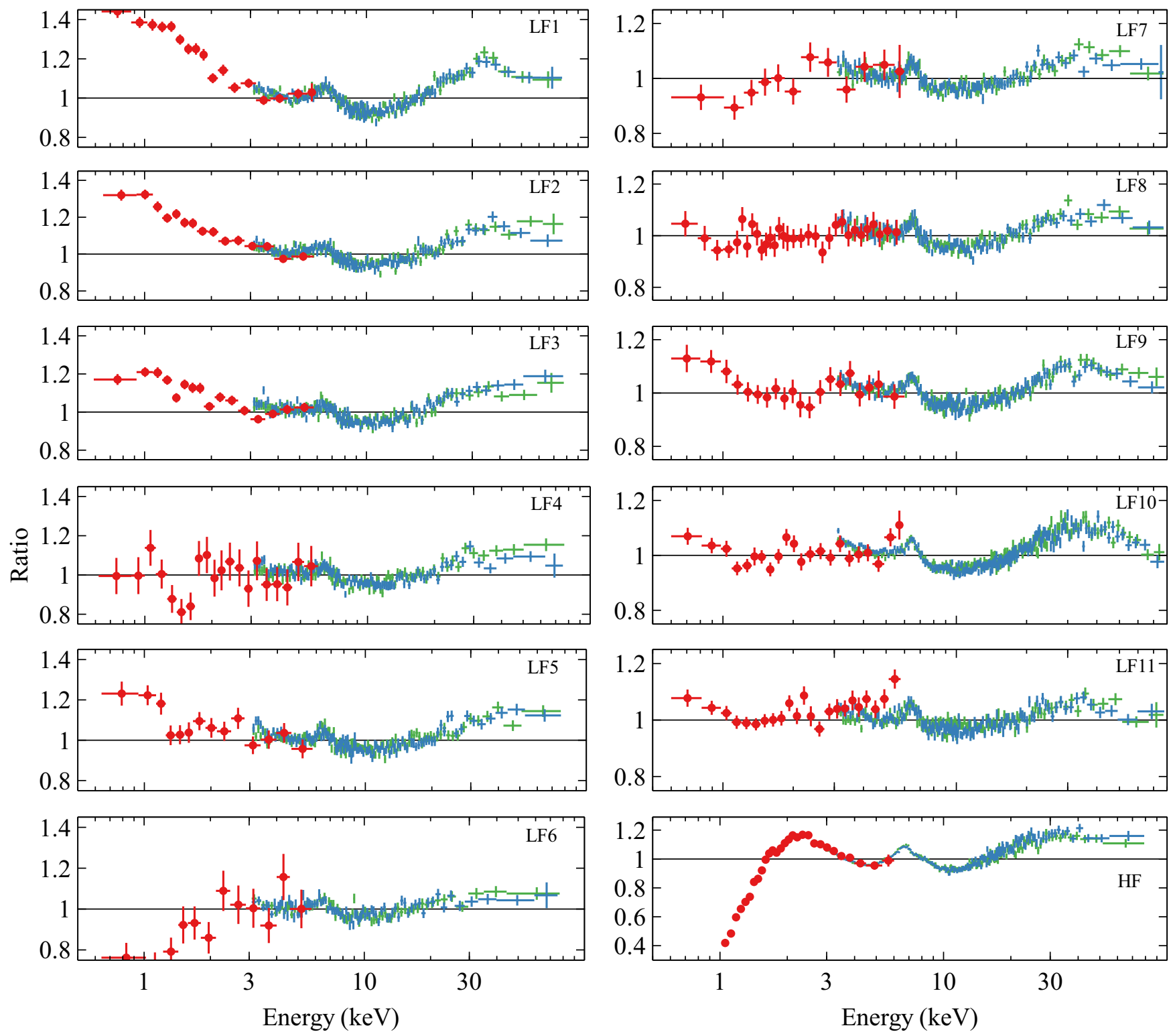

Figure 2. First 11 panels: ratio plots of GX 339-4 FPM (blue crosses: FPMA; green crosses: FPMB) and XRT (red circles) spectra fitted with a Galactic absorbed power law for LF observations in 2015 (LF1-6) and 2013 (LF7-11). Last panel: ratio plot of HF spectra fitted with a Galactic absorbed power law plus a simple blackbody component for the very HF soft state observation in 2015. All the spectra show a broad line emission feature around $6.4 \mathrm{keV}$ and a strong Compton hump above $10 \mathrm{keV}$.

spectra. A multicolour disc blackbody component diskbb is used to account for the strong disc thermal component. The full model is tbabs * (cflux*(relconv*reflionx) + cflux*reflionx + cflux*cutoffpl + diskbb) in XSPEC format. This model provides a good fit with C-stat $/ \nu=1048.68 / 971$. The best-fitting model is shown in the last panel of Fig. 6 and the best-fitting parameters are shown in the last column of Table 2. A disc density of $\log \left(n_{\mathrm{e}} / \mathrm{cm}^{-3}\right)=18.93_{-0.16}^{+0.12}$ is found in HF observations which is 100 times lower than the best-fitting value in LF observations.

So far we have obtained a good fit for the HF spectrum of GX 3394. A higher column density is required for the neutral absorber $\left(N_{\mathrm{H}}=6.2 \pm 0.2 \times 10^{21} \mathrm{~cm}^{-2}\right)$ compared to the LF observations $\left(N_{\mathrm{H}} \approx 3.4 \times 10^{21} \mathrm{~cm}^{-2}\right)$. Parker et al. (2016) obtained a higher column density of $N_{\mathrm{H}}=7.2 \pm 0.2 \times 10^{21} \mathrm{~cm}^{-2}$ for the same observation assuming $n_{\mathrm{e}}=10^{15} \mathrm{~cm}^{-3}$ for the disc. Both our result and Parker et al. (2016) are higher than the Galactic absorption column density estimated at other wavelengths (e.g. Kalberla et al. 2005), indicating a possible extra variable neutral absorber along the line of sight. Only an upper limit of the flux of the distant cold reflector is achieved $\left[\log \left(F_{\text {dis }}\right)<-10.89\right]$. The $1-10 \mathrm{keV}$ band flux values of the disc reflection component and the coronal emission increase by a factor of 13 and 6, respectively, compared to LF1. The best-fitting broad-band model shows the highest reflection fraction among all the observations considered in this work, indicating a geometry change of the disc corona system such as a small inner disc radius. A solar iron abundance $\left(1.05_{-0.15}^{+0.17}\right)$ is required for the HF spectra, which is lower than the value obtained by Parker et al. (2016), where a disc density of $n_{\mathrm{e}}=10^{15} \mathrm{~cm}^{-3}$ is assumed. 


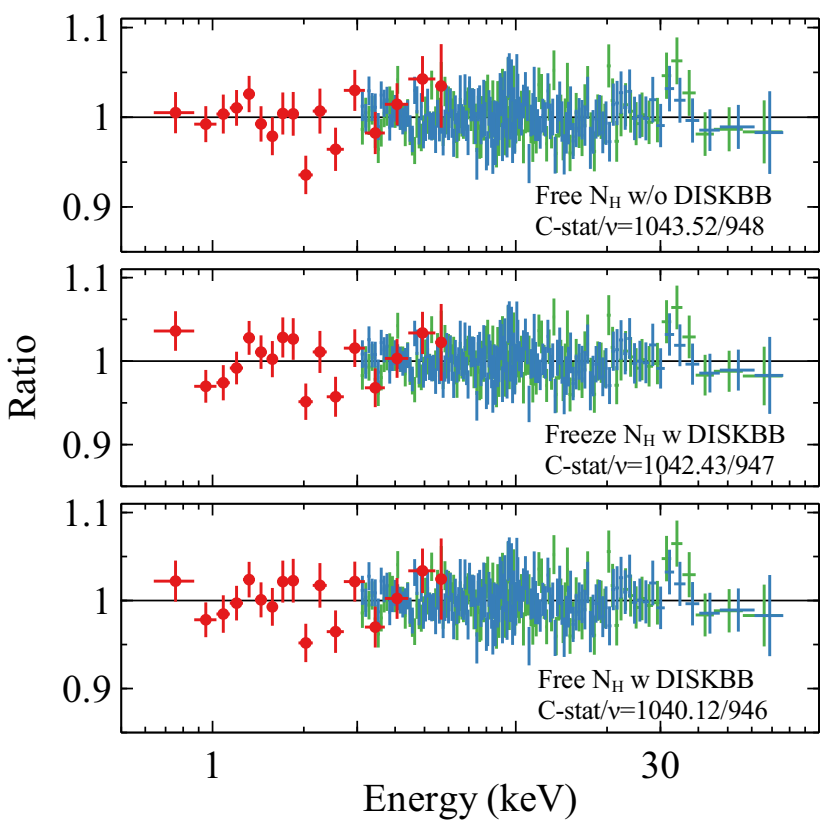

Figure 3. Ratio plots for LF1 spectra against different continuum models. Red circles: XRT; blue crosses: FPMA; green crosses: FPMB. See the text for more details.

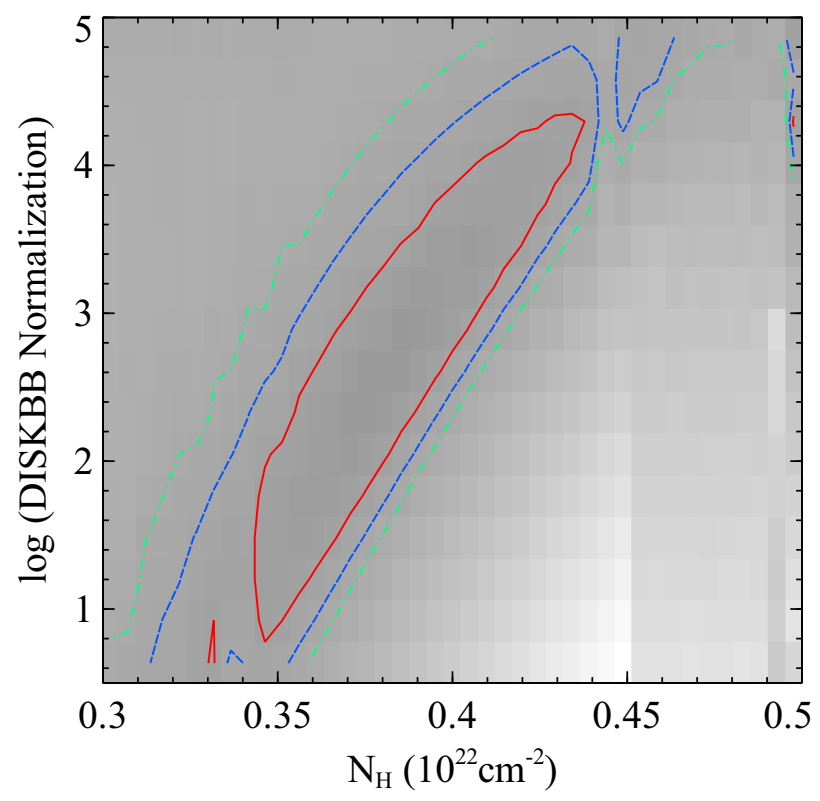

Figure 4. A contour plot of C-stat distribution on the Galactic absorption column density $N_{\mathrm{H}}$ versus diskbb model normalization parameter plane for LF1 spectra when fitted with tbabs* (diskbb+cutoffpl+relconv*reflionx+reflionx). It shows a clear degeneracy between two parameters. The lines show the $1 \sigma$ (red solid line), $2 \sigma$ (blue dashed line), and $3 \sigma$ contours (green dash-dotted line).

\section{RESULTS AND DISCUSSION}

We have obtained a good fit for the LF and the HF spectra of GX 339-4. The LF spectral modelling requires a high disc density of $\log \left(n_{\mathrm{e}} / \mathrm{cm}^{-3}\right) \approx 21$ with no additional low-temperature thermal component. The HF spectral modelling requires a 100 times lower density $\left[\log \left(n_{\mathrm{e}} / \mathrm{cm}^{-3}\right)=18.93_{-0.16}^{+0.12}\right]$ compared to LF observations.

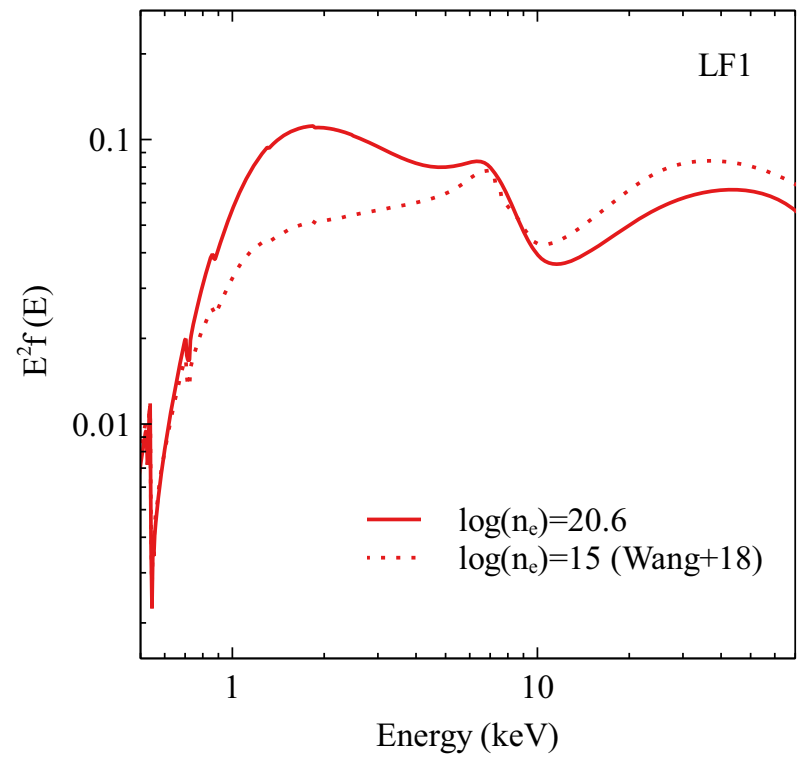

Figure 5. The best-fitting relativistic high-density reflection model for LF1 spectra (solid line) and the best-fitting relativistic reflection model obtained by Wang-Ji et al. (2018) assuming $\log \left(n_{\mathrm{e}} / \mathrm{cm}^{-3}\right)=15$ (dotted line). The plot is only shown for comparison of spectral shape. An additional di skbb component is required to fit the broad-band spectra in Wang-Ji et al. (2018).

In this section, we discuss the spectral fitting results by comparing with previous data analysis and accretion disc theories.

\subsection{Comparison with previous results}

First, the most significant difference from previous results is the close-to-solar disc iron abundance. Previously, Parker et al. (2016) and Wang-Ji et al. (2018) obtained a disc iron abundance of $Z_{\mathrm{Fe}}=$ $6.6_{-0.6}^{+0.5} Z_{\odot}$ and $Z_{\mathrm{Fe}} \approx 8 \mathrm{Z}_{\odot}$, respectively, by analysing the same spectra considered in this work. Similar result was achieved by Fürst et al. (2015). A high iron abundance of $Z_{\mathrm{Fe}}=5 \pm 1 \mathrm{Z}_{\odot}$ was also found by analysing stacked RXTE spectra (García et al. 2015). All of their work was based on the assumption for a fixed disc density $n_{\mathrm{e}}=10^{15} \mathrm{~cm}^{-3}$. By allowing the disc density $n_{\mathrm{e}}$ to vary as a free parameter during spectral fitting, we obtained a close-tosolar disc iron abundance $\left(Z_{\mathrm{Fe}}=1.50_{-0.04}^{+0.12} \mathrm{Z}_{\odot}\right.$ for LF observations and $Z_{\mathrm{Fe}}=1.05_{-0.15}^{+0.17} \mathrm{Z}_{\odot}$ for HF observations). The best-fitting disc iron abundance for the LF spectra is slightly higher than the value for the HF spectra at 90 per cent confidence. However, they are consistent within $2 \sigma$ confidence range. See the left-hand panel of Fig. 7 for the constraints on the disc iron abundance parameter. A similar conclusion was achieved by analysing the intermediate flux state spectra of Cyg X-1 (Tomsick et al. 2018) with variable density reflection model. However, a fixed solar iron abundance was assumed in their modelling.

Secondly, the best-fitting disc viewing angle measured for GX 339-4 is different in different works. The middle panel of Fig. 7 shows the constraint of the disc viewing angle given by multi-epoch LF spectral analysis and HF spectral analysis. The two measurements are consistent at the 90 per cent confidence level $\left(i=34 \pm 2 \mathrm{deg}\right.$ for the LF observations and $i=35.9_{-2.0}^{+1.6} \mathrm{deg}$ for the HF observations). Although our best-fitting value is lower compared with the measurement in Wang-Ji et al. (2018) $\left(i=40^{\circ}\right)$ and higher than the measurement in Parker et al. (2016) $\left(i=30^{\circ}\right)$, all the previous reflection based measurements are consistent with 
Table 2. The best-fitting parameters obtained by fitting (1) LF1-11 spectra simultaneously (2) only HF spectra. u: unconstrained; 1 : linked; f: fixed. $F_{\text {refl }}$ is the flux of the relativistic disc reflection component measured between 1 and $10 \mathrm{keV} ; F_{\mathrm{pl}}$ is the flux of the coronal emission measured at the same energy band; $F_{\text {dis }}$ is for the distant cold reflector. The reflection fraction $f_{\text {refl }}$ is defined as $F_{\text {refl }} / F_{\text {pl }}$ for simplicity. $L_{0.1-100 \mathrm{keV}}$ is the $0.1-100 \mathrm{keV}$ band Galactic-absorption corrected luminosity calculated using the best-fitting model. A BH mass $M_{\mathrm{BH}}=10 \mathrm{M}_{\odot}$ and a distance $d=10 \mathrm{kpc}$ are assumed.

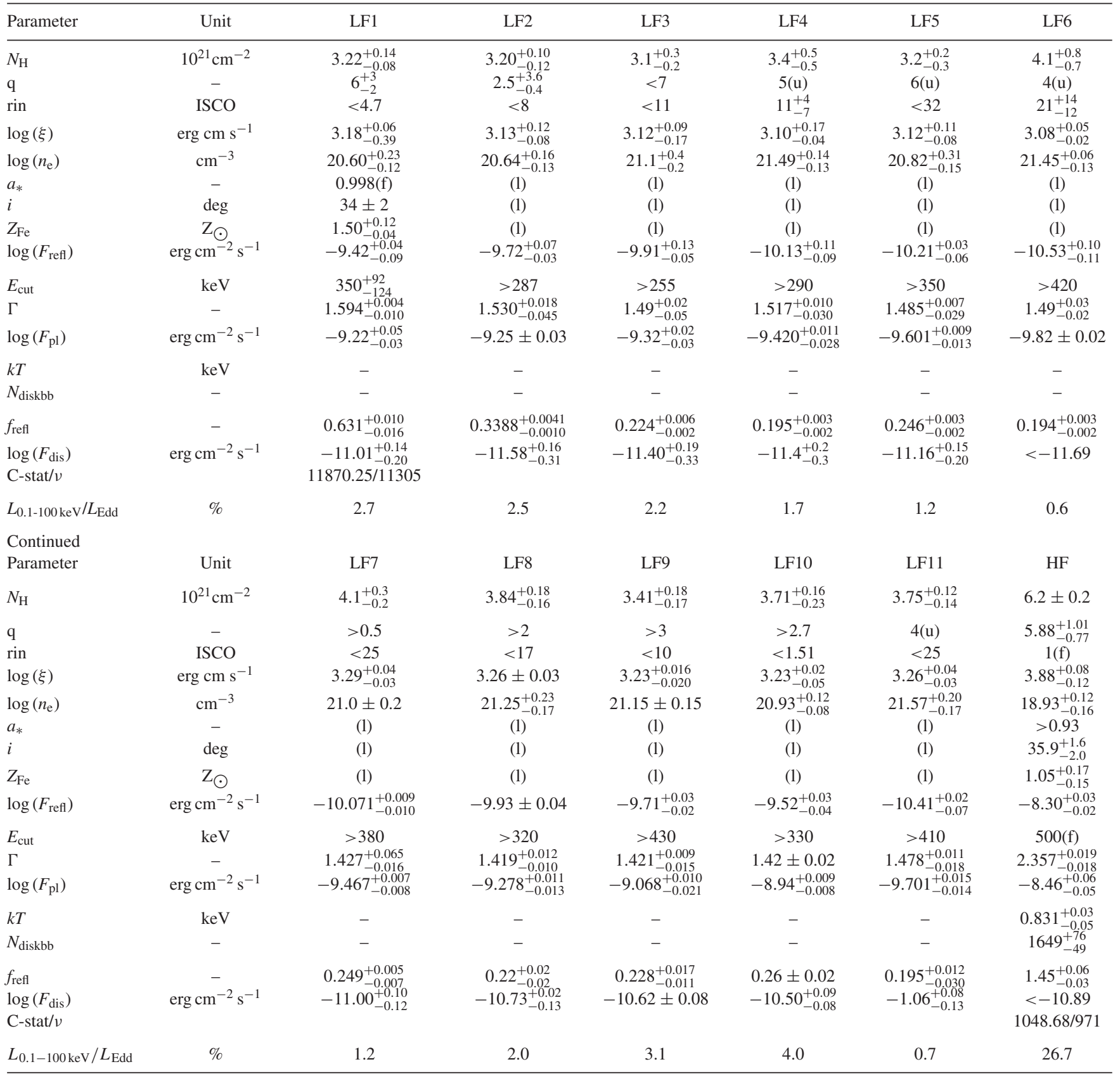

our results at $2 \sigma$ level. Similarly Tomsick et al. (2018) measured a different viewing angle for $\mathrm{Cyg}$ X-1 compared with previous works. It indicates that a slightly different viewing angle measurement might be common when allowing the disc density $n_{\mathrm{e}}$ to vary as a free parameter during the spectral fitting.

Thirdly, a high BH spin $\left(a_{*}>0.93\right)$ is given by the disc reflection modelling in the HF spectral analysis. Due to the lack of precise measurement of the source distance and the central BH mass, we can only give a rough estimation of the inner radius through the normalization of the diskbb component in the HF observations. The normalization parameter is defined as $N_{\text {diskbb }}=\left(r_{\mathrm{in}} / D_{10 \mathrm{kpc}}\right)^{2} \cos i$, where the $D_{10 \mathrm{kpc}}$ is the source distance in units of $10 \mathrm{kpc}$ and $i$ is the disc viewing angle. The best-fitting value is $N_{\text {diskbb }}=1649_{-49}^{+76}$, corresponding to an inner radius of $r_{\text {in }} \approx 45 \mathrm{~km} \approx 3 r_{g}$ assuming $M_{\mathrm{BH}}$ $=10 \mathrm{M}_{\odot}$ and $D=10 \mathrm{kpc}$. We also fitted the thermal component with kerrbb model (Li et al. 2005) as in Parker et al. (2016). kerrbb is a multicolour blackbody model for a thin disc around a Kerr BH, which includes the relativistic effects of spinning BH. The $\mathrm{BH}$ spin and the disc viewing angle are linked to the corresponding parameters in relconv. However, we found the source distance and the central $\mathrm{BH}$ mass parameters in kerrbb are not constrained during the spectral fitting. kerrbb model gives a slightly worse 

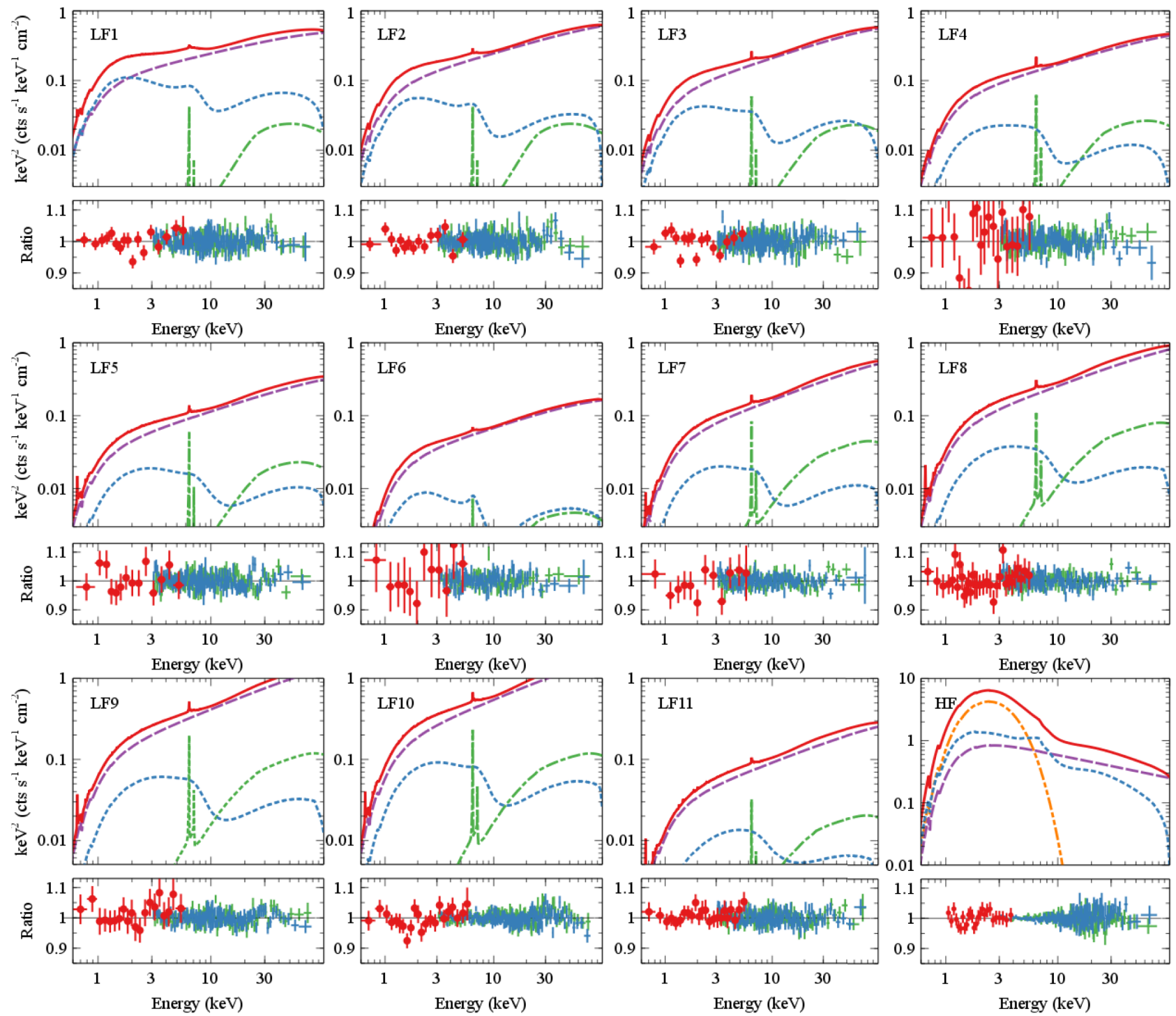

Figure 6. Top: the first 11 panels show the best-fitting models obtained by fitting LF1-11 spectra simultaneously. The last panel shows the best-fitting model obtained by fitting only HF spectra. Red solid lines: total model; blue dotted lines: relativistic reflection model; purple dashed lines: coronal emission; green dash-dotted lines: distant reflection component; orange dash-dot-dot lines: disc thermal spectrum (only needed in the HF spectral modelling). Bottom: ratio plots against the corresponding best-fitting models shown in the upper panels. Red circles: XRT; blue crosses: FPMA; green crosses: FPMB.
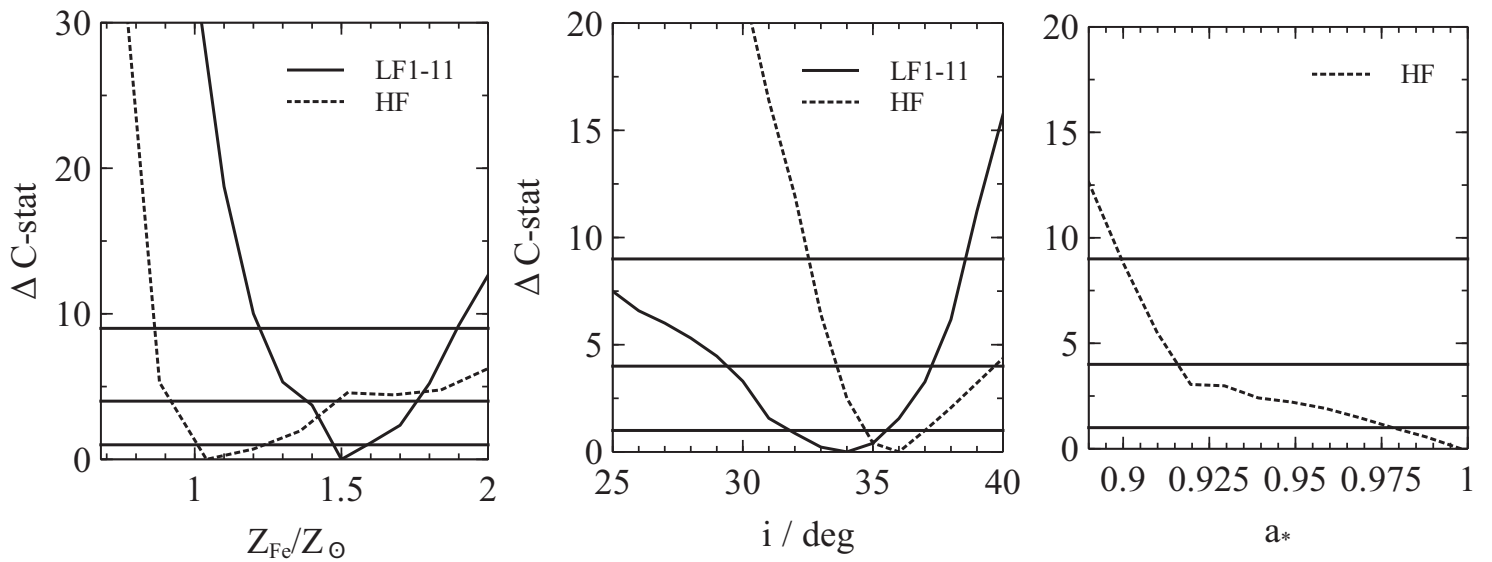

Figure 7. C-stat contour plots for the disc iron abundance and the relativistic reflection parameters obtained by fitting LF 1-11 spectra simultaneously (solid lines) and only the HF spectra (dashed line). The solid lines show the $1 \sigma, 2 \sigma$, and $3 \sigma$ ranges. 

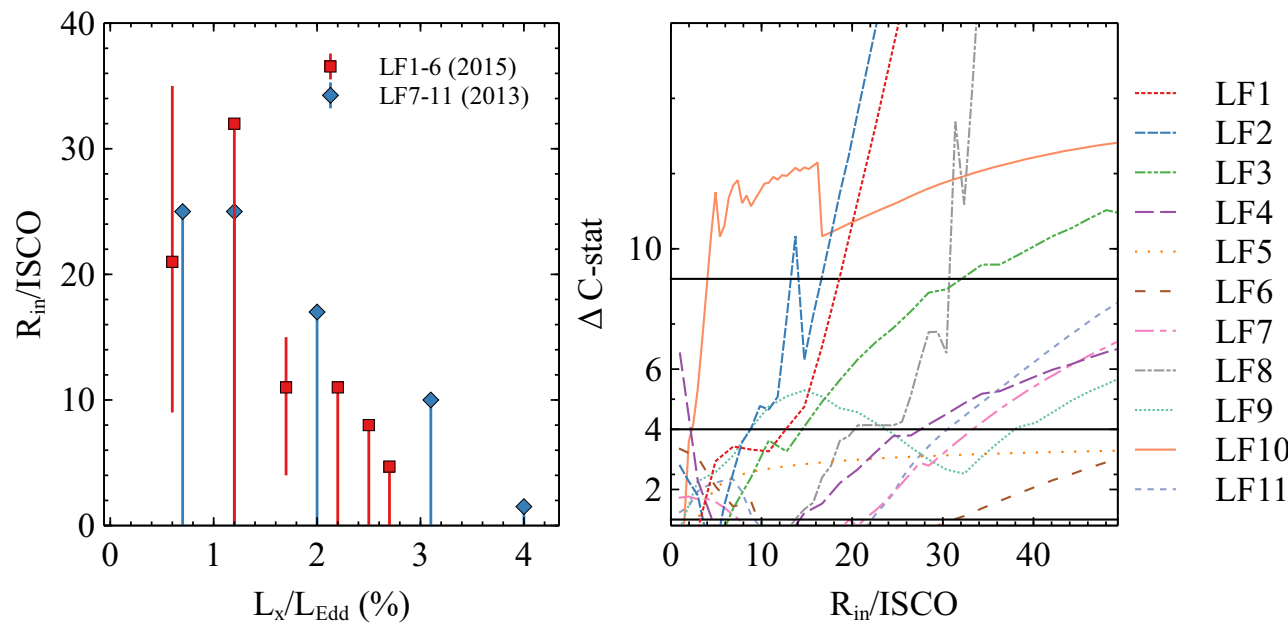

Figure 8. Left: the best-fitting inner radius of the disc versus the X-ray luminosities for LF observations. Red points represent LF1-6 observations and blue points represent LF7-11 observations. The X-ray luminosity $L_{X}$ is the $0.1-100 \mathrm{keV}$ band Galactic-absorption corrected luminosity calculated using the best-fitting model. A BH mass $M_{\mathrm{BH}}=10 \mathrm{M}_{\odot}$ and a distance $d=10 \mathrm{kpc}$ are assumed. The error bars show the 90 per cent confidence ranges of the measurements. See Table 2 for values. Right: the constraints on the inner disc radius for each LF spectra shown in different colours. The solid lines show the $1 \sigma, 2 \sigma$, and $3 \sigma$ ranges.

fit with $\Delta \mathrm{C}$-stat $\approx 7$ and 2 more free parameters compared to the diskbb model. Since the $\mathrm{BH}$ mass and distance measurement is beyond our purpose, we decide to fit the thermal spectrum in the HF observation with di skbb for simplicity. See Parker et al. (2016) for more discussion concerning the $\mathrm{BH}$ mass and the source distance measurements obtained by fitting with kerrbb. In conclusion, the high spin result in this work is obtained by modelling the relativistic disc reflection component in the HF state of GX 339-4 and consistent with previous reflection-based spectral analysis (e.g. García et al. 2015; Plant et al. 2015; Parker et al. 2016; Wang-Ji et al. 2018). Kolehmainen \& Done (2010) found an upper limit of $a_{*}$ $<0.9$ by analysing RXTE spectra. However, they assumed the disc viewing angle is the same as the binary orbital inclination, which is not necessarily the case (e.g. Tomsick et al. 2014; Walton et al. 2016).

Fourthly, there is a debate whether the disc is truncated at a significant radius in the brighter phases of the hard state. Compared with the results obtained by modelling the same spectra with $n_{\mathrm{e}}=$ $10^{15} \mathrm{~cm}^{-3}$ and an additional diskbb component (Wang-Ji et al. 2018), we find larger upper limit of the inner radius in the LF2-5 observations. For example, an upper limit of $r_{\text {in }}<8 R_{\mathrm{ISCO}}$ is obtained for the LF2 observation, larger than $r_{\text {in }}=1.8_{-0.6}^{+3.0} R_{\text {ISCO }}$ found by Wang-Ji et al. (2018). Such difference could be due to different modelling of the disc reflection component. The constraints on the inner radius $r_{\text {in }}$ are shown in the top right panel of Fig. 8. Nevertheless, we confirm that the inner radius is not as large as $r_{\text {in }}$ $\approx 100 r_{g}$ as proposed by previous analysis (e.g. Plant et al. 2015).

\subsection{High density disc reflection}

The LF and HF NUSTAR and Swift spectra of GX 339-4 can be successfully explained by high density disc reflection model with a close-to-solar iron abundance for the disc. In the LF hard states, no additional low-temperature diskbb component is require in our modelling. Instead, a quasi-blackbody emission in the soft band of the disc reflection model fits the excess below $2 \mathrm{keV}$. At higher disc density, the free-free process becomes more important in constraining low energy photons, increasing the disc surface temperature, and thus turning the reflected emission in the soft band into a quasi-blackbody spectrum. See Fig. 5 for a comparison between the best-fitting high-density reflection model for LF1 observation and a constant disc density model $\left(n_{\mathrm{e}}=10^{15}\right.$ $\left.\mathrm{cm}^{-3}\right)$

In LF states of GX 339-4, a disc density of $n_{\mathrm{e}} \approx 10^{21} \mathrm{~cm}^{-3}$ is required for the spectral fitting. Our multi-epoch spectral analysis shows tentative evidence that the disc density increases from $\log \left(n_{\mathrm{e}} / \mathrm{cm}^{-3}\right)=20.60_{-0.12}^{+0.23}$ in the highest flux state (LF1) to $\log \left(n_{\mathrm{e}} / \mathrm{cm}^{-3}\right)=21.45_{-0.13}^{+0.06}$ in the lowest flux state (LF6) during the decay of the outburst in 2015, except for LF5 observation. See Table 2 for $n_{\mathrm{e}}$ measurements. Similar pattern can be found in LF7-10 observations. In HF state of GX 339-4, we measure a disc density of $n_{\mathrm{e}} \approx 10^{19} \mathrm{~cm}^{-3}$ by fitting the broad-band spectra with a combination of high density disc model and a multicolour disc blackbody model. The disc density in HF state is 100 times lower than that in LF states. The $0.1-100 \mathrm{keV}$ band luminosity of GX 339-4 in HF state $\left(L_{X} \approx 0.28 L_{\text {Edd }}\right)$ is 10 times the same band luminosity in LF states $\left(L_{X}=0.01-0.03 L_{\text {Edd }}\right)$. While the accretion rate is rather small, the anticorrelation between the disc density and the $\mathrm{X}$-ray luminosity $\log \left(n_{\mathrm{e} 1} / n_{\mathrm{e} 2}\right) \propto-2 \log \left(L_{\mathrm{X} 1} / L_{\mathrm{X} 2}\right)$ is found to agree with the expected behaviour of a standard radiationpressure-dominated disc (e.g. Shakura \& Sunyaev 1973; Svensson \& Zdziarski 1994). See Section 4.3 for more detailed comparison between the measurements of the disc density and the predictions of the standard disc model.

\subsection{Accretion rate and disc density}

Svensson \& Zdziarski (1994, hereafter SZ94) reconsidered the standard accretion disc model (Shakura \& Sunyaev 1973, hereafter SS73) by adding one more parameter to the disc energy balance condition - a fraction of the power associated with the angular momentum transport is released from the disc to the corona, denoted as $f$. Only $1-f$ of the released accretion power is dissipated in the colder disc itself. 


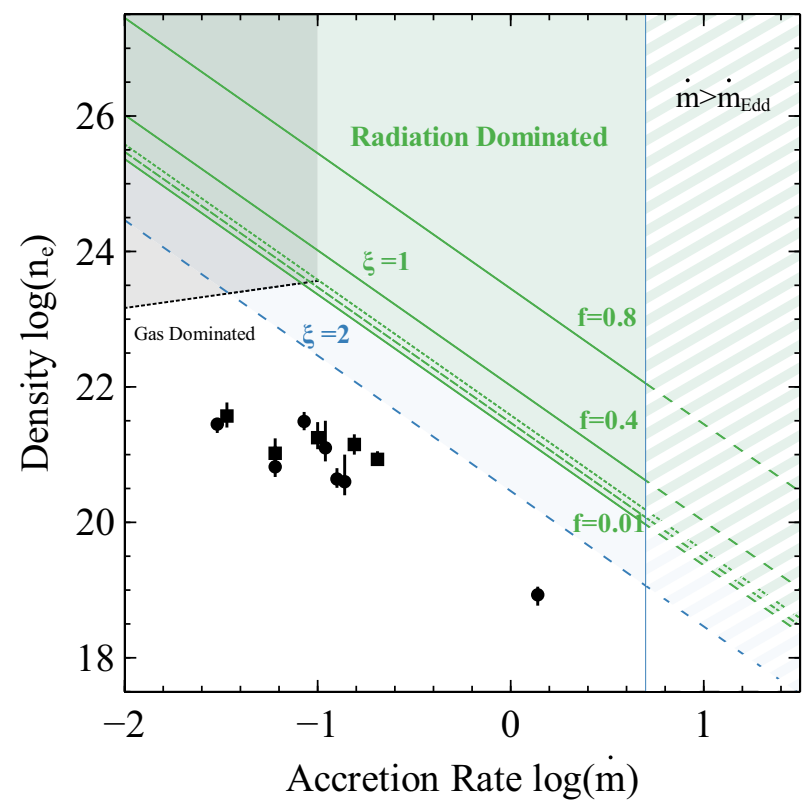

Figure 9. Disc density $\log \left(n_{\mathrm{e}} / \mathrm{cm}^{-3}\right)$ versus accretion rate $\log (\dot{m})$ based on the radiation-pressure-dominated (green) and gas-pressure dominated (black) disc solutions in SZ94, assuming $M_{\mathrm{BH}}=10 \mathrm{M}_{\odot}$ and $\alpha=0.1$. The solid green lines show the solutions with different coronal power fraction $f$ at $R=2 R_{\mathrm{S}}$. The dashed and dotted green lines show the radiation-pressuredominated solution with $f=0.01$ at $R=6,8 R_{\mathrm{S}}$, respectively. The black circular points show the surface disc density and the mass accretion rate measurements of GX 339-4 in LF and HF states in 2015 and the black squares show the measurements for observations in 2013. The mass accretion rate

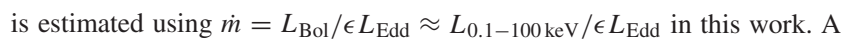
Novikov-Thorne accretion efficiency $\varepsilon=0.2$ (Novikov \& Thorne 1973; Agol \& Krolik 2000) and an inner disc radius of $R_{\text {in }}=1 R_{\mathrm{S}}$ is assumed for a spinning BH with $a_{*}=0.95$. The black vertical line shows the Eddington accretion limit $\dot{m}_{\mathrm{Edd}}=1 / \epsilon \approx 5 . \xi=1$ is assumed during the calculation as in SZ94. The blue solid line is shown for the radiation-pressure-dominated disc solution with $R=2 R_{\mathrm{S}}$ and $f=0.01$, assuming $\xi=2$.

By following SZ94, we can obtain a relation between $n_{\mathrm{e}}$ and $f$ for a radiation-pressure-dominated disc:

$n_{\mathrm{e}}=\frac{1}{\sigma_{\mathrm{T}} R_{\mathrm{S}}} \frac{256 \sqrt{2}}{27} \alpha^{-1} R^{3 / 2} \dot{m}^{-2}\left[1-\left(R_{\mathrm{in}} / R\right)^{1 / 2}\right]^{-2}[\xi(1-f)]^{-3}$,

where $\sigma_{\mathrm{T}}=6.64 \times 10^{25} \mathrm{~cm}^{2}$ is the Thomson cross-section; $R_{\mathrm{S}}$ is the Schwarzschild radius; $R$ is in the units of $R_{\mathrm{S}} ; \dot{m}$ is defined as $\dot{m}=\dot{M} c^{2} / L_{\mathrm{Edd}}=L_{\mathrm{Bol}} / \epsilon L_{\mathrm{Edd}} ; L_{\mathrm{Edd}}=4 \pi G M m_{\mathrm{p}} c / \sigma_{\mathrm{T}}=$ $2 \pi\left(m_{\mathrm{p}} / m_{\mathrm{e}}\right)\left(m_{\mathrm{e}} c^{3} / \sigma_{\mathrm{T}}\right) R_{\mathrm{S}}$ is the Eddington luminosity; $\xi$ is the conversion factor in the radiative diffusion equation and chosen to be 1,2 , or $2 / 3$ by different authors (SZ94). For a radiation-pressuredominated disc, the disc density $n_{\mathrm{e}}$ decreases with increasing accretion rate $\dot{m}$. In Fig. 9, we plot the radiation-pressure-dominated disc solutions for $\xi=1$ in green lines and a solution for $\xi=2$ in blue.

When $\dot{m}<0.1$ and $f$ approaches unity, the radiation-pressuredominated radius disappears and gas pressure starts dominates the disc. The relation between $n_{\mathrm{e}}$ and $f$ for a gas-pressure-dominated disc is

$n_{\mathrm{e}}=\frac{1}{\sigma_{\mathrm{T}} R_{\mathrm{S}}} K \alpha^{-7 / 10} R^{-33 / 20} \dot{m}^{2 / 5}\left[1-\left(R_{\text {in }} / R\right)^{1 / 2}\right]^{2 / 5}[\xi(1-f)]^{-3 / 10}$, where $K=2^{-7 / 2}\left(\frac{512 \sqrt{2} \pi^{3}}{405}\right)^{3 / 10}\left(\alpha_{\mathrm{f}} \frac{m_{\mathrm{p}}}{m_{\mathrm{e}}}\right)^{9 / 10}\left(\frac{R_{\mathrm{S}}}{r_{\mathrm{e}}}\right)^{3 / 10} . \alpha_{\mathrm{f}}$ is the finestructure constant; $m_{\mathrm{p}}$ is the proton mass; $m_{\mathrm{e}}$ is the electron mass; $r_{\mathrm{e}}$ is the classical electron radius. An example of a gas-pressuredominated disc solution for $R=2 R_{\mathrm{S}}$ and $f=0.01$ is shown by the black line in Fig. 9 for comparison. For a gas-pressure-dominated disc, the disc density increases with increasing accretion rate.

The best-fitting disc density and accretion rate values obtained by fitting GX 339-4 LF1-11 and HF spectra with high-density reflection model are shown by black points in Fig. 9. The accretion rate is calculated using $\dot{m}=L_{\mathrm{Bol}} / \epsilon L_{\mathrm{Edd}} \approx L_{0.1-100 \mathrm{keV}} / \epsilon L_{\mathrm{Edd}}$, where $\varepsilon$ is the accretion efficiency and $L_{0.1-100 \mathrm{keV}}$ is the $0.1-100 \mathrm{keV}$ band absorption corrected luminosity calculated using the best-fitting model. According to Novikov \& Thorne (1973) and Agol \& Krolik (2000), an accretion efficiency of $\epsilon=20$ per cent is assumed for a spinning BH with $a_{*}=0.95$ measured in Section 3.2. A BH mass of $10 \mathrm{M}_{\odot}$ and a source distance of $10 \mathrm{kpc}$ are assumed.

The $0.1-100 \mathrm{keV}$ band luminosity in the HF state of GX 339-4 is approximately 10 times the same band luminosity in the LF states. The disc density in the HF state is two orders of magnitude lower than the density in the LF1-6 states. The anticorrelation between its density and accretion rate is expected according to the radiationpressure-dominated disc solution in SZ94 $\left(\log \left(n_{\mathrm{e}}\right) \propto-2 \log (\dot{m})\right.$, as in equation 1$)$. However, the disc density measurements for GX 3394 are lower than the predicted values for corresponding accretion rates. See Fig. 9 for comparison between measurements and theoretical predictions in SZ94. Following are possible explanations for the mismatch: (1) The disc density shown in equation (1) is assumed to be constant throughout the vertical profile of the disc (SS73). The $n_{\mathrm{e}}$ parameter we measure using reflection spectroscopy is however the surface disc density within a small optical depth (Ross \& Fabian 2007). For example, three-dimensional MHD simulations show that the vertical structure of radiation-pressuredominated disc density is centrally concentrated (e.g. Turner 2004; Hirose, Krolik \& Stone 2006). (2) The accretion rate might be underestimated by assuming $L_{\mathrm{Bol}} \approx L_{0.1-100 \mathrm{keV}}$, although we do not expect other bands of GX 339-4 to make a large contribution to its bolometric luminosity. (3) There is a large uncertainty on the BH mass, the disc accretion efficiency, and the source distance measurements for GX 339-4. For example, the most recent nearinfrared study shows that the central BH mass in GX 339-4 could be within $2-10 \mathrm{M}_{\odot}$ (Heida et al. 2017). Nevertheless, the use of the high-density reflection model enables us to estimate the density of the disc surface in different flux states of an XRB and an anticorrelation between $n_{\mathrm{e}}$ and $L_{\mathrm{X}}$ has been found in GX 339-4.

\subsection{Future work}

In our work, we conclude that the high-density reflection model can explain both the LF and HF spectra of GX 339-4 with a close to solar iron abundance. No additional blackbody component is statically required during the spectral fitting of the LF states. On one hand, the strong degeneracy between the diskbb component and the absorber column density is due to the low $\mathrm{S} / \mathrm{N}$ of the Swift XRT observations. More pile-up free soft band spectra are required to obtain a more detailed spectral shape at the extremely LF state of GX 339-4, such as from NICER. On the other hand, more detailed spectral modelling is required. For example, a more physics model, such as Comptonization model, is required for the coronal emission modelling in the broad-band spectral analysis. The disc thermal photons from the disc to the reflection layer need to be taken into 
account in future reflection modelling, especially in the XRB soft states where a strong thermal spectrum is shown.

\section{ACKNOWLEDGEMENTS}

JJ acknowledges support by the Cambridge Trust and the Chinese Scholarship Council Joint Scholarship Programme (201604100032). DJW acknowledges support from an STFC Ernest Rutherford Fellowship. ACF acknowledges support by the ERC Advanced Grant 340442. MLP is supported by European Space Agency (ESA) Research Fellowships. JFS has been supported by NASA Einstein Fellowship grant No. PF5-160144. JAG acknowledges support from the Alexander von Humboldt Foundation. This work made use of data from the NUSTAR mission, a project led by the California Institute of Technology, managed by the Jet Propulsion Laboratory, and funded by NASA. This research has made use of the NUSTAR Data Analysis Software (NuSTARDAS) jointly developed by the ASI Science Data Center and the California Institute of Technology. This work made use of data supplied by the UK Swift Science Data Centre at the University of Leicester. We acknowledge support from European Space Astronomy Center (ESAC).

\section{REFERENCES}

Agol E., Krolik J. H., 2000, ApJ, 528, 161

Arnaud K. A., 1996, in George H. J., Jeannette B., eds, Astronomical Data Analysis Software and Systems V, A.S.P. Conference Series, Vol. 101, p. 17

Barr P., White N. E., Page C. G., 1985, MNRAS, 216, 65P

Chiang C.-Y., Walton D. J., Fabian A. C., Wilkins D. R., Gallo L. C., 2015, MNRAS, 446, 759

Dauser T., Garcia J., Wilms J., Böck M., Brenneman L. W., Falanga M., Fukumura K., Reynolds C. S., 2013, MNRAS, 430, 1694

Dauser T., García J., Walton D. J., Eikmann W., Kallman T., McClintock J., Wilms J., 2016, A\&A, 590, A76

Esin A. A., McClintock J. E., Narayan R., 1997, ApJ, 489, 865

Fabian A. C. et al., 2009, Nature, 459, 540

Fürst F. et al., 2015, ApJ, 808, 122

García J., Kallman T. R., 2010, ApJ, 718, 695

García J. A., Fabian A. C., Kallman T. R., Dauser T., Parker M. L., McClintock J. E., Steiner J. F., Wilms J., 2016, MNRAS, 462, 751

García J. A., Steiner J. F., McClintock J. E., Remillard R. A., Grinberg V., Dauser T., 2015, ApJ, 813, 84

George I. M., Fabian A. C., 1991, MNRAS, 249, 352

Gou L. et al., 2014, ApJ, 790, 29

Grupe D., Komossa S., Leighly K. M., Page K. L., 2010, ApJS, 187, 64

Heida M., Jonker P. G., Torres M. A. P., Chiavassa A., 2017, ApJ, 846, 132

Hirose S., Krolik J. H., Stone J. M., 2006, ApJ, 640, 901

Hynes R. I., Steeghs D., Casares J., Charles P. A., O'Brien K., 2003a, ApJ, 583, L95

Hynes R. I., Steeghs D., Casares J., Charles P. A., O’Brien K., 2003b, ApJ, 583, L95

Jiang J., Walton D. J., Fabian A. C., Parker M. L., 2019, MNRAS, 483, 2958

Jiang J. et al., 2018, MNRAS, 477, 3711
Kalberla P. M. W., Burton W. B., Hartmann D., Arnal E. M., Bajaja E., Morras R., Pöppel W. G. L., 2005, A\&A, 440, 775

Kerr R. P., 1963, Phys. Rev. Lett., 11, 237

Kolehmainen M., Done C., 2010, MNRAS, 406, 2206

Li L.-X., Zimmerman E. R., Narayan R., McClintock J. E., 2005, ApJS, 157,335

Matsuoka M. et al., 2009, PASJ, 61, 999

Miller J. M., Homan J., Steeghs D., Rupen M., Hunstead R. W., Wijnands R., Charles P. A., Fabian A. C., 2006, ApJ, 653, 525

Miller J. M. et al., 2004, ApJ, 606, L131

Miller J. M. et al., 2008, ApJ, 679, L113

Morrison R., McCammon D., 1983, ApJ, 270, 119

Muñoz-Darias T., Casares J., Martínez-Pais I. G., 2008, MNRAS, 385, 2205

Narayan R., 2005, Ap\&SS, 300, 177

Narayan R., McClintock J. E., 2008, New Astron. Rev., 51, 733

Novikov I. D., Thorne K. S., 1973, in Dewitt C., Dewitt B. S., eds, Black Holes (Les Astres Occlus), Gordon and Breach, New York, NY (USA), p. 343

Oda M., Gorenstein P., Gursky H., Kellogg E., Schreier E., Tananbaum H., Giacconi R., 1971, ApJ, 166, L1

Parker M. L., Miller J. M., Fabian A. C., 2018, MNRAS, 474, 1538

Parker M. L. et al., 2014, MNRAS, 443, 1723

Parker M. L. et al., 2015, ApJ, 808, 9

Parker M. L. et al., 2016, ApJ, 821, L6

Plant D. S., Fender R. P., Ponti G., Muñoz-Darias T., Coriat M., 2015, A\&A, 573, A 120

Reis R. C., Fabian A. C., Ross R. R., Miniutti G., Miller J. M., Reynolds C., 2008, MNRAS, 387, 1489

Reis R. C., Miller J. M., Reynolds M. T., Fabian A. C., Walton D. J., Cackett E., Steiner J. F., 2013, ApJ, 763, 48

Reynolds C. S., Brenneman L. W., Lohfink A. M., Trippe M. L., Miller J. M., Fabian A. C., Nowak M. A., 2012, ApJ, 755, 88

Reynolds C. S., Nowak M. A., 2003, Phys. Rep., 377, 389

Ross R. R., Fabian A. C., 2005, MNRAS, 358, 211

Ross R. R., Fabian A. C., 2007, MNRAS, 381, 1697

Shakura N. I., Sunyaev R. A., 1973, A\&A, 24, 337 (SS73)

Steiner J. F., McClintock J. E., Remillard R. A., Gou L., Yamada S., Narayan R., 2010, ApJ, 718, L117

Svensson R., Zdziarski A. A., 1994, ApJ, 436, 599 (SZ94)

Tanaka Y. et al., 1995, Nature, 375, 659

Tomsick J. A., Yamaoka K., Corbel S., Kaaret P., Kalemci E., Migliari S., 2009, ApJ, 707, L87

Tomsick J. A. et al., 2014, ApJ, 780, 78

Tomsick J. A. et al., 2018, ApJ, 855, 3

Turner N. J., 2004, ApJ, 605, L45

Walton D. J. et al., 2016, ApJ, 826, 87

Walton D. J. et al., 2017, ApJ, 839, 110

Wang-Ji J. et al., 2018, ApJ, 855, 61

Wang H., Zhou H., Yuan W., Wang T., 2012, ApJ, 751, L23

Willingale R., Starling R. L. C., Beardmore A. P., Tanvir N. R., O’Brien P. T., 2013, MNRAS, 431, 394

Wilms J., Allen A., McCray R., 2000, ApJ, 542, 914

Zdziarski A. A., Gierliński M., Mikołajewska J., Wardziński G., Smith D. M., Harmon B. A., Kitamoto S., 2004, MNRAS, 351, 791

This paper has been typeset from a $\mathrm{T}_{\mathrm{E}} \mathrm{X} / \mathrm{LT} \mathrm{E} \mathrm{X}$ file prepared by the author. 